\title{
Tunable self-injection locked green laser diode
}

\author{
Md Hosne Mobarok Shamim, ${ }^{1}$ Tien Khee Ng, ${ }^{2}$ Boon S. OoI, ${ }^{2}$ And \\ Mohammed Zahed Mustafa Khan ${ }^{1} *$
}

\author{
${ }^{1}$ Optoelectronic Research Laboratory, Department of Electrical Engineering, King Fahd University of Petroleum \& Minerals (KFUPM), Dhahran \\ 31261, Saudi Arabia \\ ${ }^{2}$ Computer, Electrical and Mathematical Sciences and Engineering (CEMSE) division, King Abdullah University of Science \& Technology \\ (KAUST), Thuwal 23955-6900, Saudi Arabia \\ *Corresponding author: zahedmk@kfupm.edu.sa
}

Received XX Month XXXX; revised XX Month, XXXX; accepted XX Month XXXX; posted XX Month XXXX (Doc. ID XXXXX); published XX Month XXXX

\begin{abstract}
We report the first employment of self-injection locking scheme for the demonstration of a tunable InGaN/GaN semiconductor laser diode. We have achieved $7.11 \mathrm{~nm}$ (521.10- 528.21 $\mathrm{nm})$ tunability in green color with different injection currents and temperatures. The system exhibited mode spectral linewidth as narrow as $\sim 69 \mathrm{pm}$ and side mode suppression ratio as high as $\sim 28 \mathrm{~dB}$, with a maximum optical power of $\sim 16.7 \mathrm{~mW}$. In the entire tuning window, extending beyond $520 \mathrm{~nm}$, a spectral linewidth of $\leq 100 \mathrm{pm}$, high power, and stable performance were consistently achieved, making this first-of-its-kind compact tunable system attractive for spectroscopy, imaging, sensing systems and visible light communication. (c) 2018 Optical Society of America
\end{abstract}

OCIS codes: (140.3520) Lasers, injection-locked; (140.5960)

Semiconductor lasers; (060.2605); Lasers, tunable (140.3600); Visible

lasers (140.7300)

http://dx.doi.org/10.1364/OL.99.099999

Green lasers are finding niche applications in bio-imaging [1], holographic displays [2], infrared spectroscopy [3], interferometric metrology, sensing, etc. However, multi-mode broad emission and inept wavelength tuning characteristics of InGaN/GaN diode based lasers pose a challenge for their practical application. Hence, a narrow linewidth lasing from these light emitters, and ability to control its wavelength, is essential, thus providing a larger degree of flexibility to the deployed systems. In this regard, distributed feedback (DFB) and distributed Bragg reflector (DBR) lasers are generally exercised to obtain a single longitudinal mode (SLM) operation, but, is living in its infancy in the visible region. Therefore, external cavity diode lasers (ECDL) are more commonly employed system in achieving tunability and near single longitudinal mode (SLM) operation utilizing a laser diode. Consequently, Chi et al. and Chen et al. demonstrated a tunable high power ( $>1 \mathrm{~W}$ ) [4] and low power $(<100 \mathrm{~mW})[5]$ laser system based on green semiconductor laser diode.

Among ECDL systems, Littrow configuration has been the most popular and widely used approach to achieve tunable SLM using a visible laser diode [4-11]. A diffraction grating structure is usually employed with a precise electronic controller, in the system, for wavelength selection and mode splitting into different diffraction orders. While the first order is feedback into the laser active region, the zeroth order mode serves as an output optical beam. Tuning ranges of $\sim 9.2 \mathrm{~nm}$ [4] and $\sim 10.5 \mathrm{~nm}$ [5] covering $508.8 \mathrm{~nm}$ to 518 $\mathrm{nm}$ and $512.53 \mathrm{~nm}$ to $523.03 \mathrm{~nm}$, respectively, and corresponding linewidths of $8 \mathrm{pm}$ and $10 \mathrm{MHz}$, have been reported. However, output beam misalignment [12], huge power loss (inefficient usable optical power) [13], the trade-off between output optical power and tuning range [4], etc., are the known limitation of this system. While the former shortcoming was later resolved in Littman-Metcalf configuration with the expense of an additional mirror to fix the output beam direction, but, resulted in additional complexity in the system. Hence, a cost-effective, compact and efficient tunable laser system is preferred in real scenarios.

Furthermore, these systems generally operate semiconductor laser diodes at lower injection currents, above the threshold, to ensure tunable and near SLM generation since the system suffers from stability and retaining SLM operation if the laser exhibits several multi-longitudinal lasing modes, which is the case at higher current injections. This poses another limitation of the system wherein the optical power generating capability of the laser diode is not fully exploited.

In this work, we propose and experimentally demonstrate employment of self-injection locking (SIL) scheme to realize a tunable laser system. The configuration is based on a partially reflective mirror and a green InGaN/GaN commercial laser diode, thus making the system simple and cost-effective. Because of the partial nature of the reflector, from which the usable output light beam exits, a small part of this light beam is optically feedback into the laser active region for locking purpose, thus ensuring fixed direction and robust operation. By controlling the distance between the laser diode and the mirror, that forms an external cavity, successful locking of various longitudinal modes is accomplished. The system is discretely tunable up to $7.11 \mathrm{~nm}$ in the green color region, and even at high injection currents. Through optimization of controlling parameters, spectral linewidths of up to $69 \mathrm{pm}$ and side mode suppression ratio (SMSR) of $28 \mathrm{~dB}$ are measured with high optical powers. To the best of our knowledge, this is the first report of self-injection locked based tunable laser system.

Fig. 1 represents the block diagram and the laboratory photograph (inset) of the self-injection locked green color tunable laser system utilizing a commercial InGaN/GaN green laser diode, exhibiting a maximum $50 \mathrm{~mW}$ optical power (Thorlabs L520P50), mounted on Thorlabs TCLDM9 laser diode mount. The output light beam from the laser facet was collimated by an aspheric lens (Thorlabs, A110TM-A) of focal length $6.24 \mathrm{~mm}$ and numerical 
aperture 0.40. A 92:8 pellicle beam-splitter (Thorlabs, BP108) was used to split $8 \%$ of the light beam into an optical spectrum analyzer (OSA, Yokogawa AQ6373B with $0.02 \mathrm{~nm}$ resolution) via a biconvex lens, L3 (Thorlabs, LB1471-A-ML) of focal length $50 \mathrm{~mm}$ for diagnostic purpose. The rest of the $92 \%$ collimated laser light beam falls on the polka-dot beam-splitter (Edmund Optics) with $70 \%$ transmissivity working as a partially reflective mirror (splitting the beam at $0^{\circ}$ angle), thus forming laser - mirror external cavity. The beam-splitter is mounted on a kinematic mount (Thorlabs, KM100) with two knobs to adjust the feedback angle and placed on a singleaxis translational stage to control the external cavity length, thus providing three degrees of freedom for fine-tuning. 30\% of the incoming light beam is feedback into the laser active region by polka-dot while the transmitted 70\% (at 'L2') is the usable optical power for various multi-disciplinary applications. Two external cavity lengths are studied in this work viz. $28 \mathrm{~cm}$ or $12 \mathrm{~cm}$.

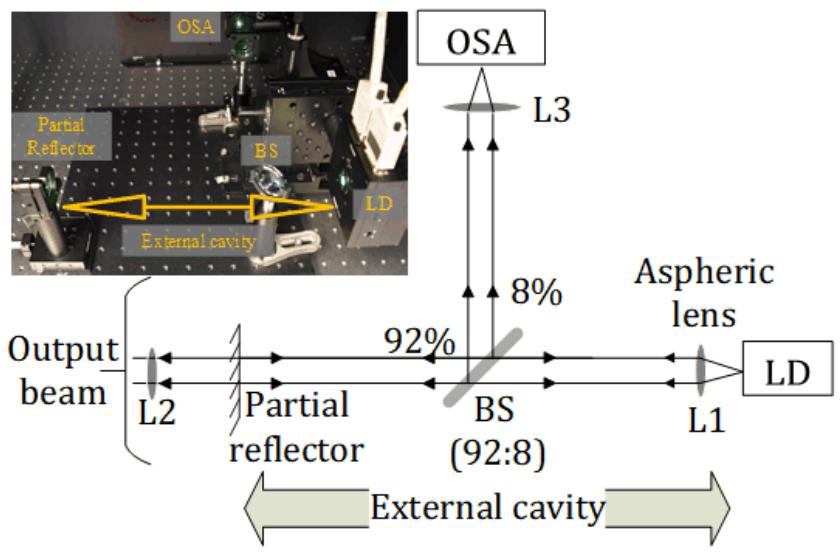

Fig. 1. Block diagram of self-injection locked tunable laser system. Laboratory photograph is shown in the inset.
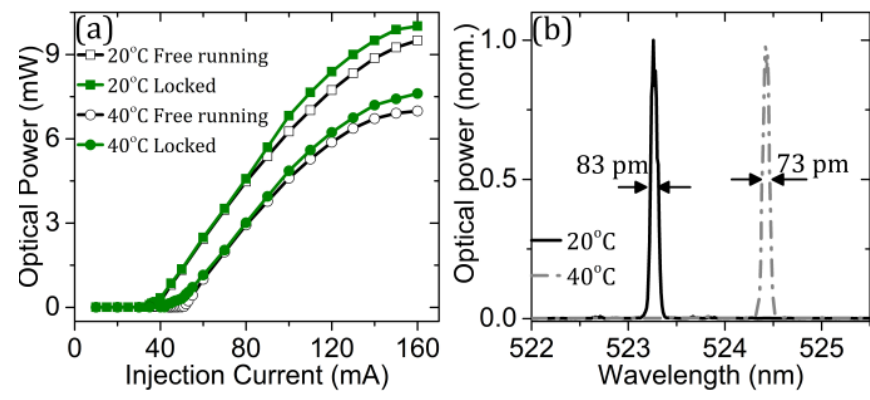

Fig. 2. (a) L-I characteristics of self-injection locked tunable laser system at 20 and $40^{\circ} \mathrm{C}$. The free-running characteristics are also shown for comparison purpose. (b) Single longitudinal lasing mode operation at $36(20)$ and $47(40) \mathrm{mA}\left({ }^{\circ} \mathrm{C}\right)$ injection currents.

Reduction in threshold current of the laser diode is a key signature of efficient self-injection locking, determined by a significant improvement of the laser optical power at a fixed injection current above the threshold, and is evident from the L-I characteristics curve of Fig. 2 (a). The threshold current is found to reduce from 40 to $34 \mathrm{~mA}$ at $20^{\circ} \mathrm{C}$ and 52 to $44 \mathrm{~mA}$ at $40^{\circ} \mathrm{C}$ heat sink temperature. This is attributed to the lower gain threshold in an
ECDL system compared to the free running laser counterpart [14]. Moreover, the slope efficiency increased from $0.09(0.075)$ to 0.11 (0.083) W/A at $20(40)^{\circ} \mathrm{C}$, thus improving the optical power, in general, by $>0.6 \mathrm{~mW}$ at higher injections, under SIL. The near SLM operation of the tunable laser system at 20 and $40^{\circ} \mathrm{C}$ at subthreshold (defined as current values below the free running and above the self-injection locked threshold currents) injection currents are presented in Fig. 2(b). Spectral linewidths of 83 and 73 $\mathrm{pm}$ are measured at $36 \mathrm{~mA}\left(20^{\circ} \mathrm{C}\right)$ and $47 \mathrm{~mA}\left(40^{\circ} \mathrm{C}\right)$ injection currents, at $28 \mathrm{~cm}$ external cavity length.
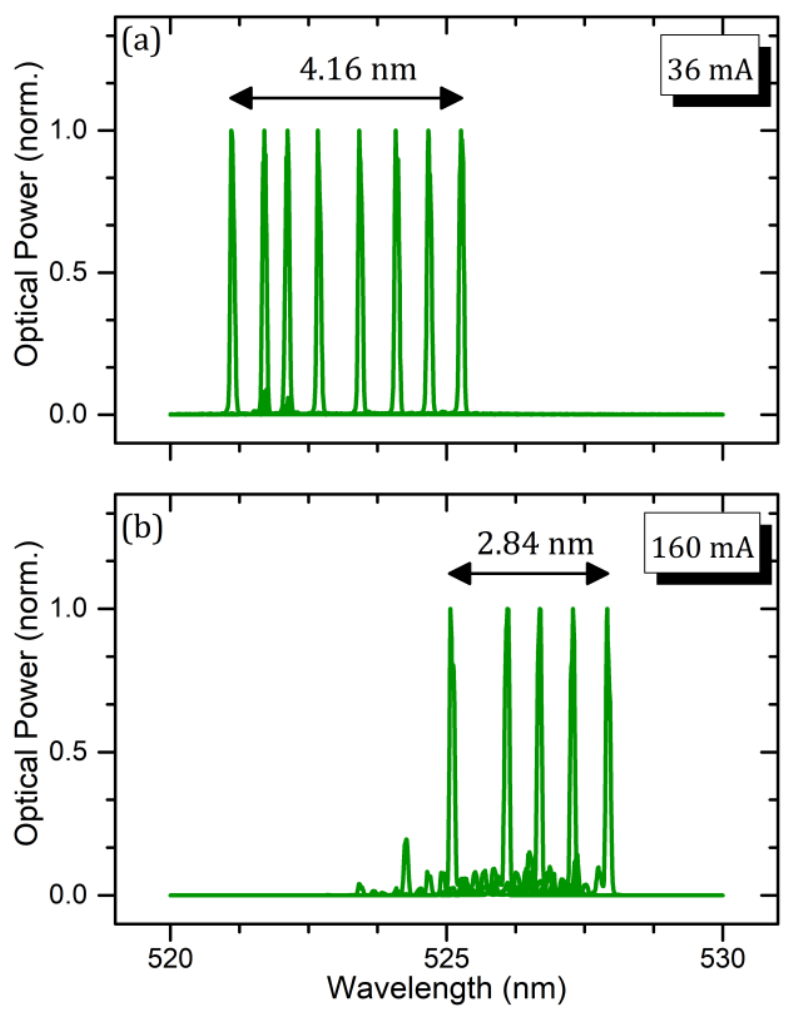

Fig. 3. The superimposed lasing spectra demonstrating the tunability of green laser diode at $20^{\circ} \mathrm{C}$, and at: (a) low injection current of $36 \mathrm{~mA}$ and (b) high injection current of $160 \mathrm{~mA}$.

Fig. 3 (a) and (b) shows an extended tunability of $4.16 \mathrm{~nm}$ $(521.10-525.26 \mathrm{~nm})$ at $36 \mathrm{~mA}$ injection current compared to 2.84 $\mathrm{nm}(525.07-527.91 \mathrm{~nm})$ at $160 \mathrm{~mA}$, at $20^{\circ} \mathrm{C}$ room temperature. Thanks to less mode competition in the lasing spectrum at lower injection compared to higher injection current, thereby exhibiting wider tuning window [5]. The tunability is accomplished by a careful control of external cavity length and feedback angle, to achieve round-trip phase-match of any particular longitudinal mode, whose wavelength (frequency) resonates within the composite cavity (i.e. laser and external cavity). This ensures selfinjection locking of that particular mode, and all the power from the neighboring side modes consolidates in increasing this dominant mode power while suppressing themselves, thus significantly improving the SMSR. It is to be noted that, although tunability was possible at all injection currents discretely, for ease in discussion, we have selected low $(36 \mathrm{~mA})$ and a high $(160 \mathrm{~mA})$ current values that collectively cover the entire demonstrated tuning window. Moreover, we also set an SMSR threshold of $10 \mathrm{~dB}$ for the system 
performance investigation throughout this work. Hence, the actual wavelength tunability is found to be considerably larger than the reported ones should a threshold SMSR of $6 \mathrm{~dB}$ be considered.
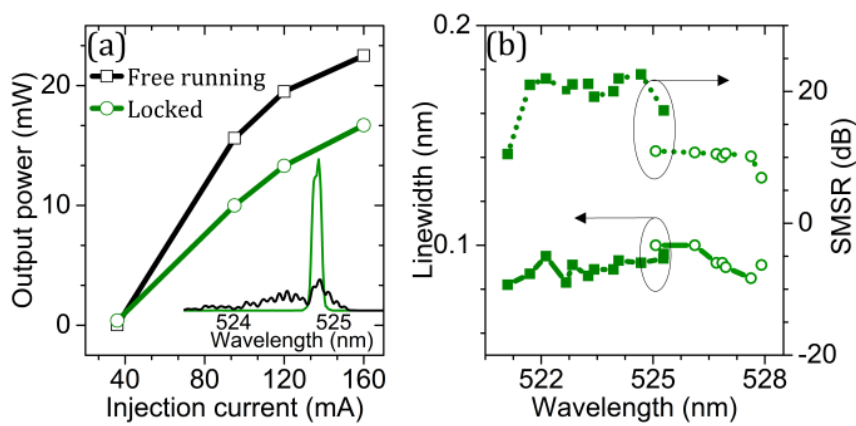

Fig. 4. (a) Comparison of the output optical power under free running and self-injection locked case with an inset showing a free running and locked case at $160 \mathrm{~mA}$. (b) Measured spectral linewidth and SMSR of the tunable locked modes at $36 \mathrm{~mA}$ (square symbols) and $160 \mathrm{~mA}$ (circle symbols) injection currents. The external cavity is fixed at $28 \mathrm{~cm}$.

The measured optical power (at ' $L 2$ ') of the system, from a tunable locked mode, is compared with the free-running case in Fig. 4(a) at different injection currents. At low current injection, power extraction efficiency of $65-70 \%$ is noted, with a maximum value of $74 \%$ at $160 \mathrm{~mA}$, corresponding to an optical power of $\sim 16.7 \mathrm{~mW}$ with $524.85 \mathrm{~nm}$ locked mode. It is worth mentioning at this point that in our SIL based tunable laser system there is no trade-off between the tunability and power efficiency, which is very common in Littrow configuration [6,7], thus enabling to achieve such high efficiency at high injection currents as well. The measured spectral linewidth and SMSR of various locked modes across the tuning window are plotted in Fig. 4(b) at both low and high injection currents. At $36 \mathrm{~mA}$ (i.e. across $4.16 \mathrm{~nm}$ tuning window), all the locked modes showed $\geq 20 \mathrm{~dB}$ SMSR, which is more than twice in value compared to $160 \mathrm{~mA}$ (i.e. $>10 \mathrm{~dB}$ ), while across the collective tuning window of $6.81 \mathrm{~nm}$, the spectral linewidth of the modes are found to be $\leq 100 \mathrm{pm}$. Lastly, we found that SMSR values, corresponding to the extreme locked mode wavelengths, at both currents, are found to decrease. This indicates operation at the extreme of the active region gain profile thus fully exploiting it.

The effect of temperature is studied in two different instances, at $20^{\circ} \mathrm{C}$ room temperature and $40^{\circ} \mathrm{C}$, and the results are plotted in Fig. 5. A tunability spanning from $521.10 \mathrm{~nm}$ to $527.91 \mathrm{~nm}$, covering $6.81 \mathrm{~nm}$ is measured at $20^{\circ} \mathrm{C}$, whereas, at $40^{\circ} \mathrm{C}$, the range of tunability is from $522.3 \mathrm{~nm}$ to $528.21 \mathrm{~nm}$, which covers $5.91 \mathrm{~nm}$, as depicted in Fig. 5(a). This red shift in the tuning window is attributed to the temperature dependent bandgap shrinkage, thereby causing redshift of the active-region gain profile. Notice that, increasing the temperature reduces the total tuning range, however, a closer look at individual injection currents contribution towards the tunability, as illustrated in the inset of Fig. 5 (a), lower and higher injection currents behave reciprocally. On increasing the temperature from $20^{\circ} \mathrm{C}$ to $40^{\circ} \mathrm{C}$, lower injection current $(36 \mathrm{~mA}$ at $20^{\circ} \mathrm{C}$ and $47 \mathrm{~mA}$ at $40^{\circ} \mathrm{C}$ ) secures higher tuning range from 4.16 to $4.73 \mathrm{~nm}$. On the contrary, high injection current of $160 \mathrm{~mA}$ experience a reduction in the tuning range from $2.84 \mathrm{~nm}$ to $2.41 \mathrm{~nm}$ on increasing the temperature. This is ascribed to wider gain profile access at the higher temperature with mere lasing mode competition at low injection current, thus extending the tuning range. On the other hand, at high injection currents, the mode competition probably intensifies and dominates at high temperature that results in narrowing of the tuning window.
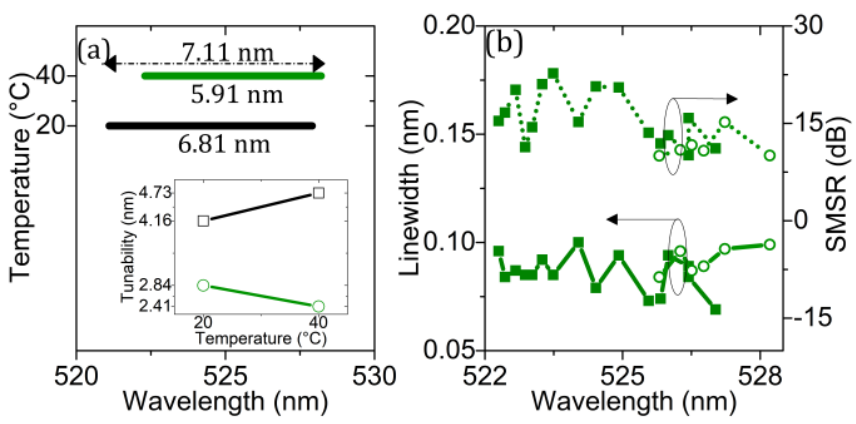

Fig. 5. (a) Collective tuning range of the system at low injection, $36 \mathrm{~mA}$ for $20^{\circ} \mathrm{C}$ and $47 \mathrm{~mA}$ for $40^{\circ} \mathrm{C}$, and high injection, $160 \mathrm{~mA}$ at $20^{\circ} \mathrm{C}$ and $40^{\circ} \mathrm{C}$. The inset shows the individual contribution of low (square symbols) and high (circle symbols) injection current towards the achieved tunability. (b) Measured spectral linewidth and SMSR across the collective tuning span of $5.91 \mathrm{~nm}$ for low (square symbols) and high (circle symbols) injection currents at $40^{\circ} \mathrm{C}$.

Fig. 5(b) summarizes the results of spectral linewidth and SMSR across the tuning range of $5.91 \mathrm{~nm}$ at $40^{\circ} \mathrm{C}$, at both injection currents. A very consistent locked modes linewidth, staying below $100 \mathrm{pm}$, is measured throughout the tuning window, with the smallest value of $69 \mathrm{pm}$ at $47 \mathrm{~mA}$ injection current. We strongly believe that inclusion of additional partial mirror based cavity, within an external cavity, would potentially reduce the modes linewidths further. On the other hand, SMSR at low (high) injection current is observed in the range of 22.7 - 10.1 (15.2 - 10.0) dB. We postulate that this fluctuation across the various locked modes is due to active-region gain profile alteration as a result of optical feedback, which could be profound at high temperature. In a nutshell, an aggregate tunability of $7.11 \mathrm{~nm}$ is measured from the system, as a function of injection current and temperature, with > $10 \mathrm{~dB}$ SMSR and $<100 \mathrm{pm}$ spectral linewidth.
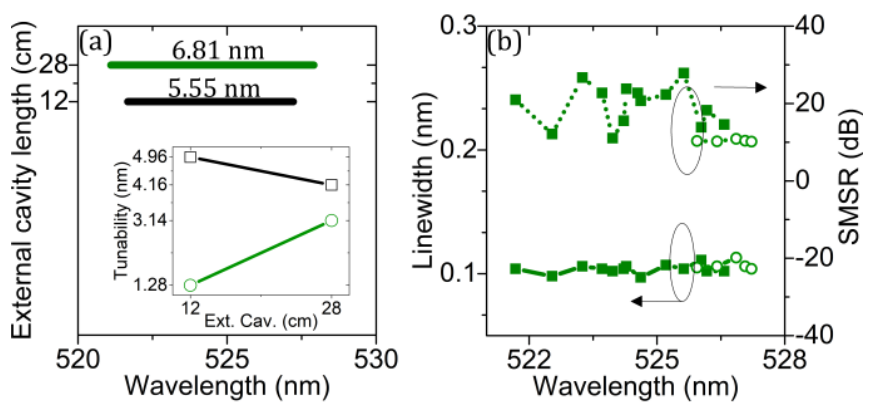

Fig. 6. (a) Collective tuning range of the system at low injection, $36 \mathrm{~mA}$, and high injection, $160 \mathrm{~mA}$, at two different external cavity lengths, and at $20^{\circ} \mathrm{C}$. The inset shows the individual contributions of low (square symbols) and high (circle symbols) towards the achieved tunability. (b) Measured spectral linewidth and SMSR across the collective tuning span of $5.55 \mathrm{~nm}$ for low (square symbols) and high (circle symbols) injection currents at $12 \mathrm{~cm}$ external cavity. 
To understand the effect of cavity length on the tuning range of the SIL based tunable laser system, we have investigated the effect of low $(36 \mathrm{~mA})$ and high $(160 \mathrm{~mA})$ injection current on a reduced $12 \mathrm{~cm}$ external cavity, and at $20^{\circ} \mathrm{C}$ room temperature. From Fig. 6 (a), we observe that a collective tunable span of the SIL laser diode reduced from $6.81 \mathrm{~nm}$ to $5.55 \mathrm{~nm}$ after reducing the external cavity from $28 \mathrm{~cm}$ to $12 \mathrm{~cm}$, respectively. However, individual tunability of the low and high injection current displayed an opposite trend compared to the inset of Fig. 5 (a). The tunability at $36 \mathrm{~mA}$ injection current is found to increases from $4.16 \mathrm{~nm}$ to $4.96 \mathrm{~nm}$ while it reduces from $3.14 \mathrm{~nm}$ to $1.28 \mathrm{~nm}$ at $160 \mathrm{~mA}$, on reducing the external cavity length from 28 to $12 \mathrm{~cm}$. This is ascribed to the higher level of difficulty in precise tuning of the tilting knobs at the shorter external cavity, which we believe can be solved using high precision motorized translational stage. Moreover, shorter cavity leads to higher power injection ratio that increases the instability in the system, thus making it more difficult to lock a near SLM with > $10 \mathrm{~dB}$ SMSR. Deployment of an appropriate high precision tuning controlling device in the reduced external cavity system should enhance the tunability performance further.
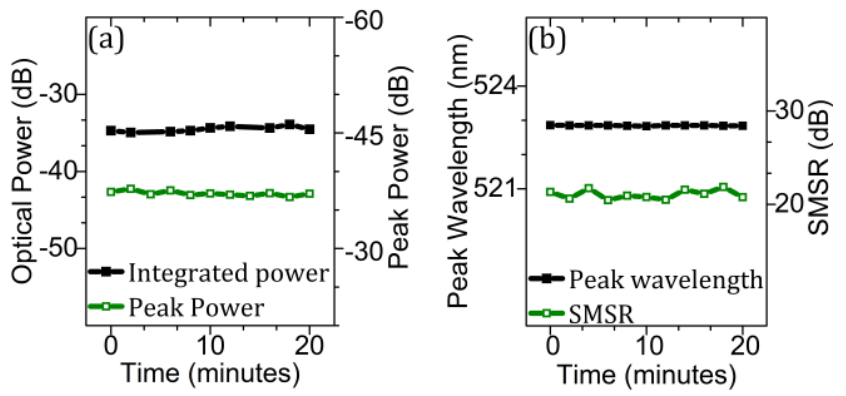

Fig. 7. Short-term stability of the self-injection locked tunable laser system showing the stability of (a) total integrated and peak optical power (measured at ' $L 3$ '), and (b) peak wavelength and SMSR, of 522.86 $\mathrm{nm}$ locked mode, at $36 \mathrm{~mA}$ injection current, $20^{\circ} \mathrm{C}$ temperature, and 28 cm external cavity.

Moreover, we have investigated the performance of the reduced external cavity tunable system by examining the measured spectral linewidth and SMSR across the tuning window of $5.55 \mathrm{~nm}$. Referring to Fig. 6(b), again, at low injection current SMSR is found to vary in the range of 27.8 to $10.2 \mathrm{~dB}$, with the former value being the best result achieved. While at high injection current, the SMSR displayed a steady average value of $\sim 10.5 \mathrm{~dB}$ across the tuning range. This observation dictates SIL being sensitive at low injection current compared to high injection current, probably due to reduced optical feedback and operation in the sub-threshold current region. Nonetheless, the observed linewidth of the tunable SIL modes across the tuning wavelengths stays close to $100 \mathrm{pm}$ at both injection currents.

Finally, we performed the short-term stability test on a tunable $522.86 \mathrm{~nm}$ locked mode at an injection current of $36 \mathrm{~mA}$ and $20^{\circ} \mathrm{C}$ for 20 minutes. The results are plotted in Fig. 7 at 2 minutes interval. The total integrated and peak optical powers are very stable throughout the time span, as can be observed from Fig. 7 (a), with a mere variation of $\sim 1 \mathrm{~dB}$ across the entire 20 minutes of the experiment. In, Fig. 7 (b), which represents the fluctuation of the corresponding mode SMSR and peak wavelength over time, is found to be steady at $\sim 20 \mathrm{~dB}$ ( $\sim 1 \mathrm{~dB}$ variation) and within a range of $522.84-522.86$ ( $\sim 20$ pm variation), respectively. Thus, superior performance is exhibited by the SIL based tunable system strengthening the scope of deploying this configuration in practical applications.

In summary, a self-injection locked based $7.11 \mathrm{~nm}$ tunable laser system is proposed and demonstrated on a green InGaN/GaN laser diode. A near single longitudinal mode tunability was observed in the entire tuning range with SMSR and spectral linewidth reaching values of $28 \mathrm{~dB}$ and $69 \mathrm{pm}$, respectively. The tunable laser system exhibited $74 \%$ power efficiency with a measured tunable locked mode optical power of $16.7 \mathrm{~mW}$. This compact and cost-effective configuration would enable a simplified tunable laser system for practical deployment in multi-disciplinary applications.

Funding. Authors acknowledge the support from Deanship of Research, King Fahd University of Petroleum and Minerals (KFUPM) (grant no. KAUST004); King Abdulaziz City for Science and Technology (KACST), (grant nos. EE2381 and KACST TIC R2FP-008); Partial support from King Abdullah University of Science and Technology (KAUST) baseline funding (grant nos. BAS/1/1614-01-01, KCR/1/2081-01-01, and GEN/1/6607-0101 ); and support from KAUST-KFUPM Special Initiative (KKI) Program (REP/1/2878-01-01).

\section{References}

1. J. Miyazaki and T. Kobayahsi, Photonics 4, 32 (2017).

2. J. Hofmann, G. Blume, D. Jedrzejczyk, B. Eppich, D. Feise, S. Kreutzmann, A. Sahm, and K. Paschke, Opt. Rev. 23, 141 (2016).

3. D. A. Kalashnikov, A. V. Paterova, S. P. Kulik, and L. A. Krivitsky, Nat. Photonics 10, 98 (2016).

4. M. Chi, O. B. Jensen, and P. M. Petersen, Opt. Lett. 41, 4154 (2016).

5. Y.-H. Chen, W.-C. Lin, H.-Z. Chen, J.-T. Shy, and H.-C. Chui, IEEE Photonics J. 9, 1 (2017).

6. M. Chi, O. B. Jensen, and P. M. Petersen, Appl. Opt. 55, 2263 (2016).

7. N. Ruhnke, a Müller, B. Eppich, M. Maiwald, B. Sumpf, G. Erbert, and G. Tränkle, Opt. Lett. 39, 3794 (2014).

8. T. Laurila, T. Joutsenoja, R. Hernberg, and M. Kuittinen, Appl. Opt. 41, 5632 (2002).

9. D. J. Lonsdale, A. P. Willis, and T. A. King, Meas. Sci. Technol. 13, 488 (2002).

10. L. Hildebrandt, R. Knispel, S. Stry, J. R. Sacher, and F. Schael, Appl. Opt. 42, 2110 (2003).

11. J. Hult, I. S. Burns, and C. F. Kaminski, Appl. Opt. 44, 3675 (2005).

12. C. J. Hawthorn, K. P. Weber, and R. E. Scholten, Rev. Sci. Instrum. 72, 4477 (2001)

13. O. J. Hong, Wenxian and Painter, (2005).

14. M. L. M. K. C. Larry, A. Coldren Scott, and W. Corzine, Diode Lasers and Photonic Integrated Circuits (2012). 


\section{References:}

1. J. Miyazaki and T. Kobayahsi, "Photothermal Microscopy for High Sensitivity and High Resolution Absorption Contrast Imaging of Biological Tissues," Photonics 4, 32 (2017).

2. J. Hofmann, G. Blume, D. Jedrzejczyk, B. Eppich, D. Feise, S. Kreutzmann, A. Sahm, and K. Paschke, "Miniaturized diode laser module emitting green light at $532 \mathrm{~nm}$ with a power of more than $900 \mathrm{~mW}$ for nextgeneration holographic displays," Opt. Rev. 23, 141-145 (2016).

3. D. A. Kalashnikov, A. V. Paterova, S. P. Kulik, and L. A. Krivitsky, "Infrared spectroscopy with visible light," Nat. Photonics 10, 98-101 (2016).

4. M. Chi, O. B. Jensen, and P. M. Petersen, "Green high-power tunable external-cavity GaN diode laser at 515 nm," Opt. Lett. 41, 4154 (2016).

5. Y.-H. Chen, W.-C. Lin, H.-Z. Chen, J.-T. Shy, and H.-C. Chui, "SingleFrequency External Cavity Green Diode Laser," IEEE Photonics J. 9, 1-7 (2017).

6. M. Chi, O. B. Jensen, and P. M. Petersen, "Tuning range and output power optimization of an external-cavity GaN diode laser at $455 \mathrm{~nm}$," Appl. Opt. 55, 2263 (2016).

7. N. Ruhnke, a Müller, B. Eppich, M. Maiwald, B. Sumpf, G. Erbert, and G. Tränkle, "400 mW external cavity diode laser with narrowband emission at $445 \mathrm{~nm} ., "$ Opt. Lett. 39, 3794-7 (2014).

8. T. Laurila, T. Joutsenoja, R. Hernberg, and M. Kuittinen, "Tunable external-cavity diode laser at $650 \mathrm{~nm}$ based on a transmission diffraction grating.," Appl. Opt. 41, 5632-5637 (2002).

9. D. J. Lonsdale, A. P. Willis, and T. A. King, "Extended tuning and singlemode operation of anti-reflection-coated InGaN violet laser diode in a Littrow cavity," Meas. Sci. Technol. 13, 488-493 (2002).

10. L. Hildebrandt, R. Knispel, S. Stry, J. R. Sacher, and F. Schael, "Antireflection-coated blue GaN laser diodes in an external cavity and Doppler-free indium absorption spectroscopy," Appl. Opt. 42, 2110 (2003).

11. J. Hult, I. S. Burns, and C. F. Kaminski, "Wide-bandwidth mode-hop-free tuning of extended-cavity GaN diode lasers," Appl. Opt. 44, 3675 (2005).

12. C. J. Hawthorn, K. P. Weber, and R. E. Scholten, "Littrow configuration tunable external cavity diode laser with fixed direction output beam," Rev. Sci. Instrum. 72, 4477-4479 (2001).

13. O. J. Hong, Wenxian and Painter, "Design and Characterization of a Littrow configuration external cavity diode laser," in (2005).

14. M. L. M. K. C. Larry, A. Coldren Scott, and W. Corzine, Diode Lasers and Photonic Integrated Circuits (2012). 